\title{
Phages and phage therapy: past, present and future
}

\author{
Nour Essa, Martina Rossitto, Ersilia Vita Fiscarelli \\ Cystic Fibrosis Diagnostic Unit, Laboratory and Pediatric Departements, Children's Hospital and Research Institute \\ Bambino Gesù, Rome, Italy
}

\begin{abstract}
Summary
Bacteriophages are viruses that infect bacteria. Since their discovery in the last century, they have proved their effectiveness in biocontrol of bacteria. In this mini-review, we provide a brief history of bacteriophages, their life cycle and classification. We also discuss the potential use of bacteriophage in clinical therapy as an alternative to antibiotics, comparing their advantages and disadvantages.
\end{abstract}

\section{Introduction}

Bacteriophages, or simply phages, as their Greek name suggests (phagein, to eat or devour, and bacterio) are viruses infecting bacteria. Defined by Sulakvelidze as "the most ubiquitous organisms on Earth", they are abundant in all environments, including water, soil and air, occupying all those habitats where bacteria thrive. Indeed, the number of phages in aquatic systems lies within the range of $10^{4}$ to $10^{8}$ virions per milliliter and about

Correspondence: Ersilia Vita Fiscarelli, Ospedale Pediatrico Bambino Gesù, Piazza di Sant'Onofrio 4, Rome, 00165, Italy.

Tel.: +390668592844

E-mail: evita.fiscarelli@opbg.net

Key words: bacteriophages, phage therapy, antibiotics.

Contributions: The authors contributed equally

Conflict of interest: The authors declare no potential conflict of interest

Funding: None

Availability of data and materials: Data are available within the text.

Ethics approval and consent to participate: The study has been approved.

Received for publication: 26 November 2019

Accepted for publication: 20 January 2020.

${ }^{\circ}$ Copyright: the Author(s), 2020

Licensee PAGEPress, Italy

Microbiologia Medica 2020; 35:8709

doi:10.4081/mm.2020.8709

This article is distributed under the terms of the Creative Commons Attribution Noncommercial License (by-nc 4.0) which permits any noncommercial use, distribution, and reproduction in any medium, provided the original author(s) and source are credited.
$10^{9}$ virions per gram in the soil, with an estimated number of $10^{31}$. $10^{32}$ phages in the world (27).

\section{History of bacteriophage and phage therapy}

The first observation of bacteriophage dated back to 1896 . It was the British chemist Ernest H. Hankin who first reported the presence of an antimicrobial activity in the Jumna and Ganges rivers, in India (27). However, it required 30 years for the scientific community to properly investigate phages. In 1915, Frederick Twort was the first to hypothesize that nonpathogenic viruses growing on bacteria were responsible for the transparent, glassy areas he observed in bacterial culture.

Still, the discovery of phages is officially attributed to the French-Canadian Félix d'Herelle who observed the same phenomenon of bacterial lysis two years later and coined the term bacteriophages. Contrary to Twort, who seemed to favor the notion that lysis was determined by an enzyme secreted by the bacteria itself, d'Herelle was quite certain that the phenomenon he observed was due to a virus capable of parasitizing bacteria. He had to wait until the year 1939, when the newly invented electron microscope confirmed the phage viral nature.

D'Herelle was also the first who developed the idea of "phage therapy', pursuing the application of phages as therapeutic and prophylactic treatment in humans, exploiting phage selectivity towards pathogenic bacteria and investigating safety towards human host cells.

D'Hérelle founded the Bacteriophage Laboratory in France and began the production of the first commercial phage cocktails in what later became the great French company L'Oréal. At the same time, bacteriophages were also used for therapeutic purposes in the United States.

With the discovery of penicillin in 1940, the era of antibiotics started and phage therapy was abandoned in Western European Countries and North America. Nevertheless, phages continued to be used therapeutically in Eastern Europe and in the former Soviet Union states such as Poland and Georgia. In these countries, different institutions involved in the research and production of therapeutic bacteriophages were established. In particular, the Eliava Institute of Bacteriophages, Microbiology and Virology (EIBMV) of the Georgian Academy of Sciences (Tbilisi, Georgia) and the Institute Hirszfeld of Immunology and Experimental Therapy (HIIET) of the Polish Academy of Sciences (27).

\section{Phage life cycle}

Like other viruses, bacteriophages are obligate intracellular parasites. Phages, in order to reproduce, must come into contact 
with a susceptible host capable of allowing replication. Based on their infectious cycle, the vast majority of phages can be distinguished into lytic or lysogenic. Lytic phages (also named virulent phages) carry out the lytic cycle in which the virus adsorbs to the surface of target bacteria, injects its genome into the host cytoplasm and induces the bacterial molecular system to produce virions that will eventually destroy the bacterial cell liberating themselves in the surrounding environment. This cycle will continue as long as the target bacteria are present, releasing hundreds of new virions within minutes or hours (12).

Lysogenic phages (also named temperate phages) are viruses that can adopt a lysogenic cycle, as an alternative to the lytic cycle. In the lysogenic cycle, phages integrate their genome in the host nucleic acid (or eventually linger as a plasmid) assuming the quiescent state of prophage, which guarantees the viral reproduction within the bacterial cells. In response to specific stimuli, the prophage will exit from its dormant state and enter into the lytic cycle. During viral genome excision, transduction may happen, resulting in horizontal gene transfer within the bacterial population (for example that of resistance determinants). For this reason, temperate phages are not suitable for phage therapy $(7,18)$. Potentially, transduction may occur also with obligate lytic phages; however, the likelihood is very low, given the rapid killing of bacterial host and the simultaneous increase of phage number. In addition to these two types, another kind of life cycle was observed in filamentous phages in which bacteriophages kill their host without lysing it (21).

\section{Bacteriophage classification}

The classification of bacteriophages is subject to continuous discussions and debates, particularly with the increasing volume of available genomic and proteomic data. Currently, both genomic and morphological information is used in their classification. The genetic material of phages consists of double-stranded (ds) or single-stranded (ss) DNA or RNA, and their genome sizes can range from very simple (e.g. $-3.5 \mathrm{~kb}$ ssRNA genome in phage MS2) to highly complex (e.g. -500 kb dsDNA genome in Bacillus phage G) and can include modified nucleotides as protection against restriction enzymes. Morphologically, phages can be tailed ( $96 \%$ of phages), polyhedral, filamentous or pleomorphic, and some have lipid or lipoprotein envelopes. Most characterized phages belong to the Caudovirales order (ds DNA genome with a tailed morphology), divided into the three families: Myoviridae with contractile tail (for example, phage T4), Siphoviridae which possess non contractile tail (for example, phage $\lambda$ ) and Podoviridae which have very short tail (for example, phage T7) (3).

\section{Advantages and disadvantages of phage}

When compared to antibiotics, phages show several appealing properties that make their therapeutic use advisable. Table 1 summarizes some advantages and disadvantages of phages, and compares phage safety, specificity, bactericidal effect and ability to infect bacteria resident in biofilm with antibiotics.

A major problem that drawback phage application is the emergence of resistant bacteria which hamper the effectiveness of such therapy. Actually, bacteria can encounter phages using different strategies, including blocking phage adsorption or DNA entry into the bacterial cell or restriction-modification and CRISPR-Cas systems that are able to cut phage nucleic acids once they have been injected into the bacterial cell (15).

Even though bacteria could become resistant to phages, phage resistance is not nearly as worrisome as drug resistance. Like bacteria, phages mutate and can therefore evolve into counter phage-resistant bacteria (25). In addition, it appears that bacteriophage force a clinically relevant trade-off, during which organisms evolve one trait that improves fitness (a relative reproduction or survival advantage) while simultaneously suffering reduced performance in another trait. Therefore, phage therapy could be used as an 'evolutionary-based strategy' in which phages drive MDR bacteria to evolve resistance to them while recovering sensitivity to chemical antibiotics (6). Moreover, phage can be used in combination with antibiotics, as long as their mechanism of action does not interfere with phage infection and replication (16).

\section{Phage therapy in $21^{\text {st }}$ century}

Antibiotic resistance, the hot topic of $21^{\text {st }}$ century, leads to a renewed interest for phage therapy also in the Western world. In 2012, the widespread diffusion of multidrug-resistant (MDR) bacteria led the World Health Organization (WHO) to acknowledge a state of emergency all around the world, warning the possibility of entering an era where antibiotics lose their power over bacterial infections. In 2017, WHO published a list of bacteria urgently requiring new antibiotics, in order to guide and promote new antibiotics research and development; among the alternative approaches suggested, the use of clinical products containing bacteriophages was considered (26).

Over the last decades, phages have been applied to treat a great variety of bacterial infections. In particular, phage safety and efficacy have been investigated in animal models of both acute and chronic infection: for example, phages have been therapeutically used against abscesses and subcutaneous infections in mice, chronic otitis in dogs, chronic infected wounds in diabetic rats and pigs, cystic fibrosis lung infection in mice and cystic fibrosis related infection in larvae and zebra fish, mastitis in cows, osteomyelitis in rabbit $(2,4,8,23)$. In clinical human practice, phage therapy has been used for the treatment of longstanding, persistent, or chronic bacterial infections. Patients with abscesses, osteomyelitis, prostatitis, urinary tract infections, otitis, skin ulcers, venous leg and diabetic food, bed sores, suppurative fistulas and cystic fibrosis have been treated with phages, typically as last resort, when their bacterial infection did not respond to conventional treatments (2). The news of these days is of a 15year-old patient with cystic fibrosis with a disseminated Mycobacterium abscessus infection following bilateral lung transplantation; this infection has been resolved by intravenous administration of engineered phage (20). Bacteriophage has also been used successfully in the treatment of a 68-year-old diabetic patient with necrotizing pancreatitis complicated by an MDR Acinetobacter baumannii infection (24).

Besides human therapy, bacteriophages have been applied in different fields, such as food bio-preservation and disinfection of medical devices. In the context of food safety, bacteriophages can be used at different stages of foodstuff production (from farm to fork) to: i) improve animal health (phage therapy), ii) decontaminate fresh-food and ready-to-eat products, iii) disinfect food-contact surfaces. Phages and their proteins were applied successfully against several pathogenic bacteria and their biofilms including Escherichia coli, Bacillus spp., Salmonella spp., Campylobacter spp., Vibrio spp., Clostridium spp., Listeria spp., Staphylococcus spp., and Pseudomonas spp (11). Lytic bacteriophages prove to be effective also in the treatment and formation prevention of bacterial biofilms 
Table 1. Advantages and disadvantages of phage therapy versus antibiotic therapy.

\section{Phage pros}

The omnipresence of bacteriophages in the environment implies a constant exposure of humans to phages, so that phages are not xenobiotic to our bodies. Our healthy microbiome includes a virome that is, in fact, largely phagome.

Bacteriophages administered for therapeutic purposes are well tolerated by patients (13).

Phages are highly specific, with most of them infecting only a single bacterial species or even a estrict number of strains within a single species. This allows phages to target only pathogenic bacteria without disturbing the resident bacterial flora, earning them the epithet of "magic bullet" (13).

Phages are antibacterial agents that grow exponentially in numbers, at the site of infection, where the host is present.

\section{Phage cons}

It has been demonstrated that bacteriophage therapeutic use could generate some immunological response (highly dependent on the way of administration). This can be attributed to endotoxin released from the bacteria in which phages are propagated; therefore, phage formulated products need to be highly purified (13).

\section{Phage strict specificity may pose a great problem} for introducing phage therapy into clinical practice when facing variation and fast adaptation among bacteria (19).

\section{Bacteriophage pharmacology and pharmacokinetic} can be relatively complex because of i) phage dimension and life cycle characteristics (i.e. adsorption rate latency time and burst size), ii) filtering organ activity, that can rapidly clear phage from circulation, iii) phage destruction caused by gut digestive enzymes $(13,19)$

Obligatory lytic phages are bactericide able to infect the persister "dormant" cells populating the inner layer of biofilms, remaining dormant within them, and re-activating when they become metabolically active. Some phages express enzyme depolymerizing biofilm matrix constituent (13).
Extracellular polymers of biofilm matrix may slow phage penetration into bacterial surfaces. It has been suggested that, by slowing phage propagation within biofilms, bacteria may be able to escape from biofilms via standard dissemination-initiating mechanisms. Phage entrapment in the extracellular matrix and phage inactivation are also possible (10).

\section{Antibiotics cons}

Antibiotics have several side effects.

Many chemical antibiotics tend to have broad spectrums of activity, target both pathogens and normal flora of patients, disrupting natural microflora and possibly causing secondary infections or superinfections (16).

Antibiotics travel throughout the body and do not concentrate at the site of infection (16).

Certain antibiotics, that are bacteriostatic, do not kill bacteria. Antibiotics, requiring metabolically active cells, cannot exert their effect on persister cells. Biofilm bacteria can display up to 1,000-fold higher resistance to antibiotic than their planktonic counterpart $(1,17)$. commonly associated with infections of indwelling urological devices and catheter-associated urinary tract infections (5).

\section{Example of clinical trail}

Although bacteriophages were first used almost 100 years ago to treat infections, they were ignored in the Western world after the discovery of antibiotics. In other countries, however, such as Georgia and Poland, the use of phages for both preventive and therapeutic purposes continued (27).

Scientific reports on phage therapy in Eastern Europe include studies that do not always meet the criteria of modern evidencebased medicine (lack of control groups, ethics committee, etc.). For this reason, we will focus only on studies after the 1980 s conducted in accordance with the Western regulation, in which treated patients suffered from antibiotic-resistant infections.

It is noteworthy to report the study of Wright et al., 2009 (28) which is the first double-blind phase I/ II controlled clinical trial. In this study, bacteriophage cocktail was used to treat chronic otitis associated with Pseudomonas aeruginosa.

Recently, a number of clinical trials have been registered. For example, we can mention phase I safety set out in USA to treat venous leg ulcers infected by Pseudomonas aeruginosa, Staphylococcus aureus and Escherichia coli (22). Another example, the study of Jault et al.,2018 (14), which has been conducted in double blind phase using a cocktail of 12 bacteriophages to treat burn wounds clinically infected with $P$. aeruginosa.

\section{Conclusions}

We can distinguish four periods in the history of phage therapy: enthusiasm, skepticism, abandonment, and then a recent revival.

Based on clinical results, phage therapy seems to represent a promising alternative approach to antibiotics toward combating pathogenic bacteria.

However, advances in phage therapy need more robust evidence of clinical trials. Additional data are also required, such as bacteriophage formulation, dosing, efficacy and its effect on human immune response.

\section{References}

1. Abedon ST. Phage 'delay' toward enhancing bacterial escape from biofilms: a more comprehensive way of viewing resistance to bacteriophages. AIMS Microbiol 2017;3:186-227.

2. Abedon ST. Use of phage therapy to treat long-standing, persistent, or chronic bacterial infections. Adv Drug Deliv Rev 2018;145:18-35.

3. Ackermann HW. Bacteriophage taxonomy. Microbiol Aust 2011;32:90-4.

4. Cafora M. Deforian G, Forti F, et al. Phage therapy against Pseudomonas aeruginosa infections in a cystic fibrosis zebrafish model. Nature 2019;9:1528.

5. Carson L, Gorman S, Gilmore B. The use of lytic 
bacteriophages in the prevention and eradication of biofilms of Proteus mirabilis and Escherichia coli. FEMS Immunol Med Microbiol 2010;59:447-55.

6. Chan B, Sistrom M, Wertz J, et al. Phage selection restores antibiotic sensitivity in MDR Pseudomonas aeruginosa. Sci Rep 2016;6:27717.

7. Debarbieux L. La phagothérapie expérimentale à l'aube du XXI siècle. Med Mal Infect 2008;38:421-5.

8. Forti F, Roach DR, Cafora M, et al. Design of a broad-range bacteriophage cocktail that reduces Pseudomonas aeruginosa biofilms and treats acute infections in two animal models. Antimicrob. Agents Chemother 2018:62:e02573-17.

9. Furfaro LL, Payne MS, Chang BJ. Bacteriophage therapy: Clinical trials and regulatory hurdles. Front Cell Infect Microbiol 2018;8:376.

10. Gonzalez S, Fernandez L, Gutierrez D, et al. Analysis of different parameters affecting diffusion, propagation and survival of Staphylophages in bacterial biofilms. Front Microbiol 2018;9:2348.

11. Gutiérrez D, Rodríguez-Rubio L, Martínez B, et al. Bacteriophages as weapons against bacterial biofilms in the food industry. Front Microbiol 2016;7:825-40.

12. Harper D, Parracho H, Walker J, et al. Bacteriophages and biofilms. Antibiotics 2014;3:280-4.

13. Hyman P, Abedon ST. Bacteriophages in Health and Disease; CABI Press: Wallingford, UK; 2012.

14. Jault P, Leclerc T, Jennes $S$, et al. Efficacy and tolerability of a cocktail of bacteriophages to treat burn wounds infected by Pseudomonas aeruginosa (PhagoBurn): a randomised, controlled, double-blind phase $1 / 2$ trial. Lancet Infect Dis 2018;19:35-45.

15. Labrie SJ, Samson JE, Moineau S. Bacteriophage resistance mechanisms. Nat Rev Microbiol 2010;8:317-28.

16. Loc-Carrillo C, Abedon ST. Pros and cons of phage therapy. Bacteriophage 2011;1:111-4.

17. Mah TF, O'Toole GA. Mechanisms of biofilm resistance to antimicrobial agents. Trends Microbiol 2001;9:34-9.
18. Matsuzaki S, Rashel M, Uchiyama J, et al. Bacteriophage therapy: a revitalized therapy against bacterial infectious diseases. J Infect Chemother 2005;11:211-9.

19. Nilsson AS. Phage Therapy-Constraints and Possibilities. Ups J Med Sci 2014;119:192-8.

20. Osmani M, Sotello D. Alvarez S, et al. Mycobacterium abscessus infections in lung transplants: 15-year experience from a single institution. Transpl Infect Dis 2018;20:1-8.

21. Rakonjac J, Bennett N, Spagnuolo J, et al. Filamentous bacteriophage: biology, phage display and nanotechnology applications. Curr Issues Mol Biol 2018;13:51-76.

22. Rhoads DD, Wolcott RD, Kuskowski MA, et al. Bacteriophage therapy of venous leg ulcers in humans: results of a phase I safety trial. J Wound Care 2009;18:237-8.

23. Rossitto M, Fiscarelli EV, Rosati P. Challenges and promises for planning future clinical research into bacteriophage therapy against Pseudomonas aeruginosa in cystic fibrosis. An argumentative review. Front Microbiol 2018;9:775.

24. Schooley RT, Biswas B, Gill JJ. Development and use of personalized bacteriophage-based therapeutic cocktails to treat a patient with a disseminated resistant Acinetobacter baumannii infection. Antimicrob Agents Chemother 2017;61:e00954-17.

25. Semler DD, Lynch KH, Dennis JJ. The promise of bacteriophage therapy for Burkholderia cepacia complex respiratory infections. Front Cell Inf Microbiol 2012;1:28.

26. Tacconelli E, Magrini N, Kahlmeter G, Singh N. Global priority list of antibiotic-resistant bacteria to guide research, discovery, and development of new antibiotics. World Health Organization, 2017; 28.

27. Wittebole X, De Roock S, Opal SM. A historical overview of bacteriophage therapy as an alternative to antibiotics for the treatment of bacterial pathogens. Virulence 2014;5:227-35.

28. Wright A, Hawkins CH, Anggard EE, Harper DR. A controlled clinical trial of a therapeutic bacteriophage preparation in chronic otitis due to antibiotic-resistant Pseudomonas aeruginosa; a preliminary report of efficacy. Clin Otolaryngol 2009;34:349-57. 